\title{
SURFACE SEGREGATION AS A MEANS OF GETTERING Cu IN LIQUID-PHASE-EPITAXY SILICON THIN LAYERS GROWN FROM AI-Cu-Si SOLUTIONS
}

\author{
T.H. Wang, T.F. Ciszek, R. Reedy, S. Asher, and D. King \\ National Renewable Energy Laboratory, Golden, CO 80401
}

\begin{abstract}
We demonstrate that, by using the natural surface segregation phenomenon, $\mathrm{Cu}$ can be gettered to the surface from the bulk of silicon layers so that its concentrations in the liquid-phase-epitaxy (LPE) layers are much lower than its solubility at the layer growth temperature and the reported $10^{17} \mathrm{~cm}^{-3}$ degradation threshold for solar-cell performance. Secondary-ion mass spectroscopy (SIMS) analysis indicates that, within a micron-deep sub-surface region, $\mathrm{Cu}$ accumulates even in as-grown LPE samples. Slower cooling after growth to room temperature enhances this $\mathrm{Cu}$ enrichment. $X$-ray photoelectron spectroscopy (XPS) measurement shows as much as $3.2 \% \mathrm{Cu}$ in a surface region of about $50 \AA$. More surface-sensitive, ion-scattering spectroscopy (ISS) analysis further reveals about $7 \%$ of $\mathrm{Cu}$ at the top surface. These results translate to an areal getfering capacity of about $1.0 \times 10^{16} \mathrm{~cm}^{-2}$, which is higher than the available total-area density of $\mathrm{Cu}$ in the layer and substrate $(3.6 \times$ $1015 \mathrm{~cm}^{-2}$ for a uniform $1.2 \times 10^{17} \mathrm{~cm}^{-3} \mathrm{Cu}$ throughout the layer and substrate with a total thickness of $300 \mu \mathrm{m}$ ).
\end{abstract}

\section{INTRODUCTION}

Cu-Al has been found to be a good solvent system to grow macroscopically smooth Si layers with thicknesses in tens of microns on cast metallurgical-grade (MG) Si substrates by liquid-phase epitaxy (LPE) at temperatures near $900{ }^{\circ} \mathrm{C}$ [1]. This solvent system uses Al to ensure good wetting between the solution and substrate by removing silicon native oxides, and it uses $\mathrm{Cu}$ to control Al-doping into the layers. Isotropic growth is achieved because of a high concentration of solute silicon in the solution and the resulting microscopically rough interface. The incorporation of $\mathrm{Cu}$ in the $\mathrm{Si}$ layers, however, was a concern because $\mathrm{Cu}$ is a major solution component and is generally regarded as a bad impurity for silicon devices due to its fast diffusivity and deep energy levels in the bandgap. One study[2] shows that $\mathrm{Cu}$ nonetheless will not degrade solar-cell performance until above a level of $10^{17} \mathrm{~cm}^{-3}$. This threshold is expected to be even higher for thin- layer silicon solar cells owing to the less-stringent requirement on minority-carrier diffusion length. But to ensure long-term stability of solar cells, lower $\mathrm{Cu}$ concentrations in the thin layers are still preferred.

When grown under near-equilibrium conditions from a Cu-Si melt, the silicon crystal is expected to be saturated with $\mathrm{Cu}$ to its solid solubility limit at the growth temperature $\left(1.2 \times 10^{17} \mathrm{~cm}^{-3}\right.$ for $\left.900^{\circ} \mathrm{C}\right)$. More components in the melt will slightly change $\mathrm{Cu}$ incorporation about this level because of the change in free energy in the melt. Using Al-Cu-Si solutions, $\mathrm{Cu}$ incorporation will be a little less from experiments and calculation with the multi-component regular solution model. During post-growth cool-down, the $\mathrm{Cu}$ will become supersaturated and segregate to the surface or precipitate at the defect sites. The free-silicon surface is the preferred escape site for the supersaturated $\mathrm{Cu}$ atoms because the high free energy in the bulk will be spent on creating a new Cu-terminated surface if $\mathrm{Cu}$ has lower surface energy than silicon. This indeed is the case: $\mathrm{Cu}$ aggregates to the surface during cool- down after layer growth. We may incorporate this surface segregation phenomenon to effectively getter fast-diffusing $\mathrm{Cu}$ from the bulk of silicon without using a dedicated gettering procedure.

\section{LPE GROWTH OF SILICON}

The liquid phase epitaxial growth setup and procedure is described elsewhere [1]. Thin silicon layers of $10-30-\mu \mathrm{m}$ thickness were grown at temperatures around $900{ }^{\circ} \mathrm{C}$ on pure Czochralski(CZ) single crystal substrates in this study. The solution compositions were kept nearly constant at $23 \% \mathrm{Si}-28 \% \mathrm{Al}-49 \% \mathrm{Cu}$ for the growth runs to minimize their effects on $\mathrm{Cu}$ incorporation in solid silicon. After a sample is withdrawn from the solution, a free silicon surface is exposed and the sample temperature is gradually reduced to room temperature. It is during this cool-down period that surface segregation occurs for various impurities in silicon crystals (both the layer and substrate) depending on their relative surface energy to silicon.

\section{SURFACE SEGREGATION}

Surface segregation is a redistribution of solute atoms (e.g., $\mathrm{Cu}$ ) between the surface and bulk of a (Si) 
crystal from the as-grown uniform distribution until the total energy of the crystal becomes a minimum. The equilibrium surface composition (mole fracti on in the surface monolayer), $x_{c u}^{\phi}$, of $\mathrm{Cu}$ as a function of temperature is described by the Bragg-Williams equation [3]. The equation predicts a full surface coverage at temperatures somewhere below the growth temperature,

$$
\frac{x_{C u}^{\varphi}}{1-x_{C u}^{\varphi}}=\frac{x_{C u}^{B}}{1-x_{C u}^{B}} \exp \left[\frac{\Delta G+2 \Omega_{S C u}^{S}\left(x_{C u}^{\varphi}-x_{C u}^{B}\right)}{R T}\right] \text {, }
$$

where $\Delta G=\left(\mu_{S i}{ }^{O S}-\mu_{S i}{ }^{O B}\right)-\left(\mu_{\mathrm{Cu}}{ }^{D \Phi}-\mu_{\mathrm{Cu}}{ }^{O B}\right), \mu^{\prime}$ s are the surface and bulk chemical potential of pure $\mathrm{Si}$ and pure $\mathrm{Cu}, x^{B}{ }_{C u}$ is the bulk mole fraction of $\mathrm{Cu}$, and $\Omega^{s}{ }_{s i c u}$ is the interaction parameter between $\mathrm{Si}$ and $\mathrm{Cu}$ in the solid. For the grown $\mathrm{Si}$ crystal, a binary Si-Cu consideration (excluding Al) leads to $\Omega_{\text {sicu }}^{s}=12.81 R T_{\theta}$, where $T_{\theta}$ is the growth temperature.

Due to the residual stress near the surface region even in as-grown layers, segregation to the surface is not limited to the top monolayer. Thus, combined with the fast diffusivity and relatively low solubility of $\mathrm{Cu}$ in silicon, this surface segregation effect may be used to effectively getter $\mathrm{Cu}$ from the bulk of the silicon thin layers and substrates.

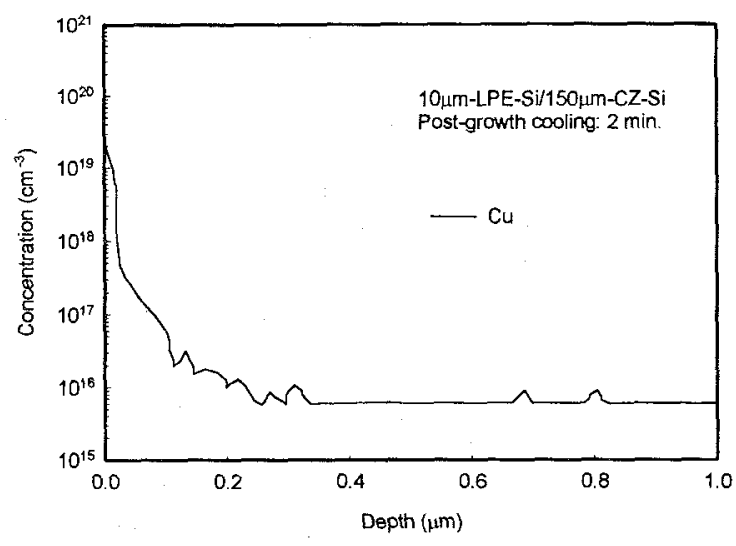

Fig.1. SIMS depth profile of $\mathrm{Cu}$ with a total sample thickness $\approx 160 \mu \mathrm{m}$.

The significance of surface segregation is evidenced by the depth profiling of $\mathrm{Cu}$ obtained with secondary-ion mass spectroscopy (SIMS) analysis in thinlayer silicon grown on high-purity single-crystal silicon substrates (Figs. 1 and 2). Because of the dynamic nature of SIMS measurements, the signal in the first $100 \AA$ is not accurate, but both samples show Cu enrichment in the $0.3-0.4-\mu \mathrm{m}$ surface region. One can easily notice the difference in the bulk $\mathrm{Cu}$ concentrations between the two samples. A logical explanation is the difference in total $\mathrm{Cu}$ content due to different substrate thicknesses. Both samples are expected to be saturated with $\mathrm{Cu}$ at the growth temperature of $900^{\circ} \mathrm{C}$. The sample in Fig. 2 is thicker and would gather more $\mathrm{Cu}$ during growth from indiffusion of $\mathrm{Cu}$ than the sample in Fig. 1. During the sample cool-down period after growth, Cu out-diffuses to the surface and results in different levels of reduction in bulk $\mathrm{Cu}$ concentrations.

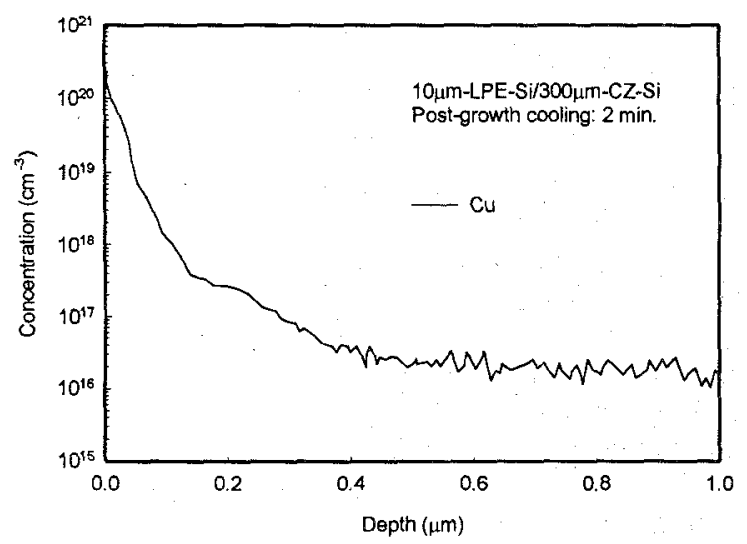

Fig.2. SIMS depth profile of $\mathrm{Cu}$ with a total sample thickness $\approx 310 \mu \mathrm{m}$.

\section{GETTERING OF Cu}

To fully use this Cu sink near the surface, a slower cooling after layer growth can be done to speed up the segregation process (Fig. 3). The same sample was analyzed with $X$-ray photoelectron spectroscopy (XPS), which shows as much as $3.2 \%$ of $\mathrm{Cu}$ in a surface region of about $50 \AA$. More surface-sensitive, ion-scattering spectroscopy (ISS) analysis reveais about $7 \% \mathrm{Cu}$ at the top surface as shown in Fig. 4. Surface contaminants (e.g., O, C) prevented accurate measurement at the top monolayer, but this composition represents the minimum amount of $\mathrm{Cu}$ at the surface.

The slow diffusers (like B shown in Fig. 3) also exhibit surface segregation, yet the effects were limited to much smaller regions, indicating that surface segregation for slow-diffusing impurities is farther away from reaching the equilibrium state during the sample cooling period. Otherwise, one would expect it to extend to a depth and exhibit a profile similar to $\mathrm{Cu}$.

According to the Bragg-Williams equation, the amount of $\mathrm{Cu}$ segregating to the surface would follow the curves in Fig. 5, where $\Delta G$ is the driving force arising from the difference between the chemical potential differentials

(surface to bulk) of pure $\mathrm{Si}$ and $\mathrm{Cu}$, and $\Omega$ is the interaction parameter (regular solution model) between $\mathrm{Si}$ and $\mathrm{Cu}$ in the solid silicon, which we determined 
experimentally to be $12.81 R T_{e}$. Therefore, it is straightforward to apply surface segregation as a gettering step following the LPE growth process.

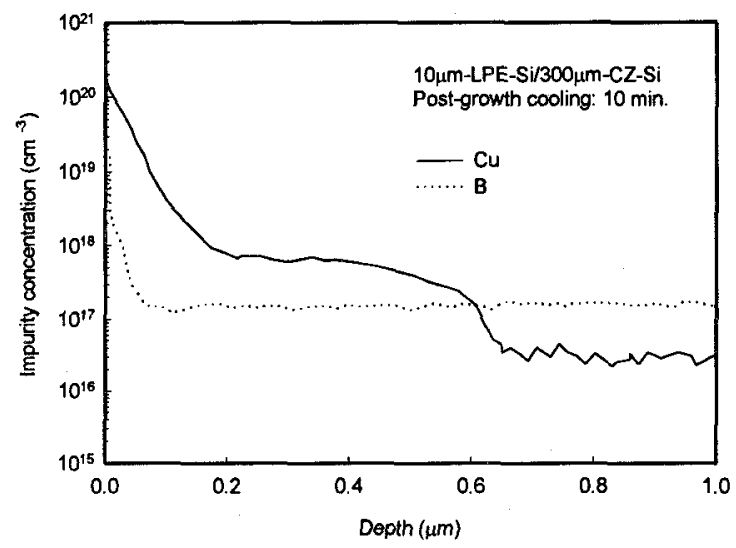

Fig. 3. Slower post-growth cooling as compared to Fig. 2.

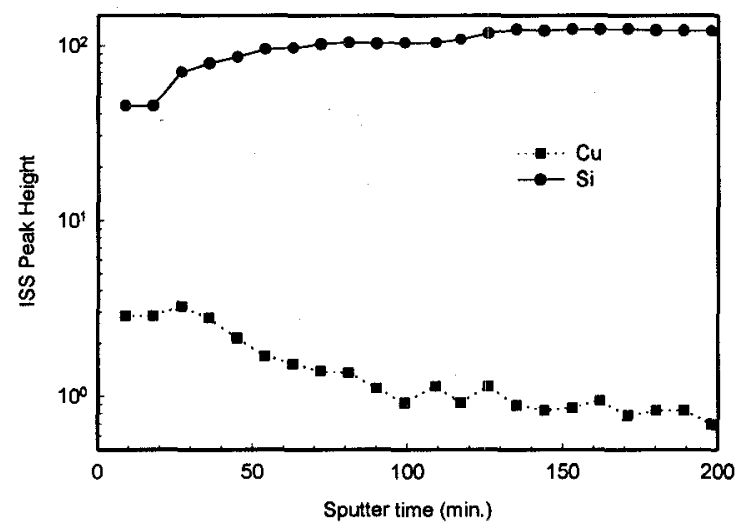

Fig.4. Cu depth profile by ISS.

Taking the $\mathrm{Cu}$ depth profiles in Fig. 3 and Fig. 4 as nearly equilibrium, the gettering capacity is estimated to be about $1.0 \times 10^{16} \mathrm{~cm}^{-2}$, higher than a monolayer of $\mathrm{Cu}$, owing to the extensiveness of $\mathrm{Cu}$ enrichment in the sub-surface region.

The total areal density of $\mathrm{Cu}$ in the layer and substrate is estimated to be $3.5 \times 10^{15} \mathrm{~cm}^{-2}$ at the uniform concentration of $1.2 \times 10^{17}$ for the total thickness of $300 \mu \mathrm{m}$, for example.

After removing the top Cu-enriched surface region by wet chemical etch, bulk concentration of $\mathrm{Cu}$ is typically about $1 \times 10^{16} \mathrm{~cm}^{-3}$, as seen in Fig. 6 . Diagnostic solar cells made in such layers exhibit efficiencies as high as $15.3 \%$, compared to $15.7 \%$ for Czochralski (CZ)-Si control cells.

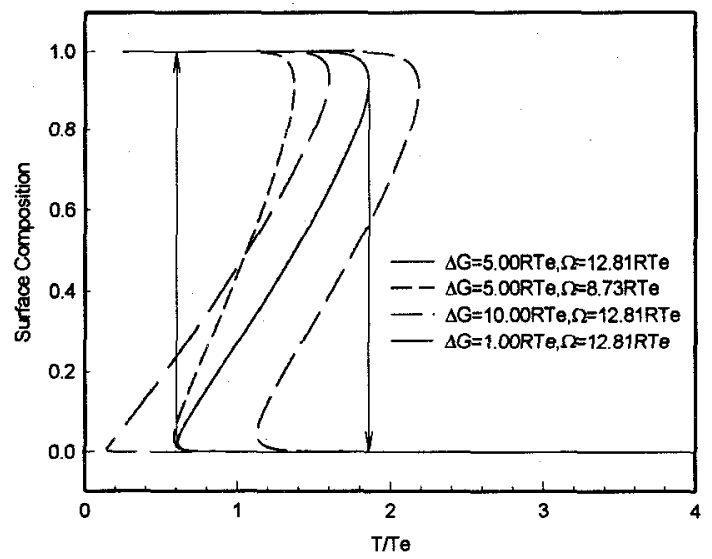

Fig.5. Equilibrium surface composition of $\mathrm{Cu}$ as a function of temperature. The upward and downward arrows are example paths for decreasing and increasing temperatures, respectively.

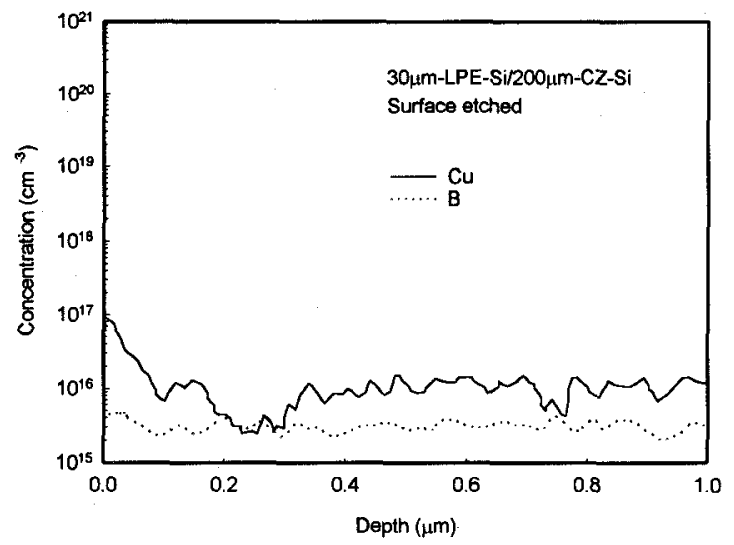

Fig.6. $\mathrm{Cu}$ depth profile after a surface etch in $3: 1: 2$ mixture of nitric, hydrofluoric, and acetic acids. The boron profile is shown as a reference to indicate no significant surface segregation between the time of the acid etch and SIMS measurement.

Note that this surface segregation will have the same effect in gettering other fast-diffusing impurities like $\mathrm{Ni}$ or $\mathrm{Fe}$. Due to their overall lower solubility in silicon $(5 \times$ $10^{16} \mathrm{~cm}^{-3}$ for $\mathrm{Ni}, 2.5 \times 10^{15} \mathrm{~cm}^{-3}$ for $\mathrm{Fe}$ at $900^{\circ} \mathrm{C}$ ), the bulk concentrations of these impurities after surface segregation should be low enough to not cause any device performance degradation, even if a low-purity metallurgical-grade-Si is used as LPE substrates. Slow diffusing impurities in a metallurgical-grade-Si substrate will not catch up the epitaxial growth front, thus they will not be of a concern. 
For grain boundaries, the relative (to the bulk) chemical potentials of $\mathrm{Si}$ and $\mathrm{Cu}$ atoms at these locations are very likely to be lower than that of a free surface. This implies that the difference between the relative grain boundary energy of $\mathrm{Si}$ and that of $\mathrm{Cu}$ is smaller than the difference between the relative surface energy of $\mathrm{Si}$ and $\mathrm{Cu}$. The same argument may be made for other defects in silicon. Experimental evidences are abundant, including SIMS analysis in which no significant impurity enrichment at grain boundaries was observed and the necessity of a thick sample in order to Cu-decorate defects in silicon [4]. Therefore, free silicon surface is the preferred escape site for fast diffusing impurities even for defected materials provided a small silicon thickness and sufficient time are given.

\section{SUMMARY}

Surface segregation can be used to effectively getter $\mathrm{Cu}$ and other fast-diffusing impurities (supposedly having lower surface energy than silicon) from bulk silicon when the impurity concentration exceeds its roomtemperature solubility and when the silicon crystal is in thin (a few hundred microns) forms. When a free-silicon surface is available, this gettering process does not need special procedures, but only a prolonged cool-down step. The estimated total areal gettering capacity of $1.0 \times 10^{16}$ $\mathrm{cm}^{-2}$ should be sufficient for $\mathrm{Cu}$ concentration up to $1.2 \times 10^{17} \mathrm{~cm}^{-3}$ in a silicon wafer as thick as $800 \mu \mathrm{m}$.

Silicon layers grown by LPE from Al-Cu solutions have shown below $1 \times 10^{16} \mathrm{~cm}^{-3}$ in $\mathrm{Cu}$ concentration, and such a level of $\mathrm{Cu}$ impurity does not appear to degrade solar-cell performances.

This gettering procedure may be conveniently incorporated in many (sheet or ribbon) crystal growth and wafer processes, by using a slow cool-down and a surface-removal procedure. As a matter of fact, such a procedure may have already existed in many current processes naturally.

\section{ACKNOWLEDGMENTS}

We thank Dr. Y.S. Tsuo for diagnostic cell fabrications. Support for this work was provided by the U.S. Department of Energy under contract No. DE-AC3683CH10093 to the National Renewable Energy Laboratory.

\section{REFERENCES}

[1] T.H. Wang and T.F. Ciszek, Conference Record of the Twenty-Fourth IEEE Photovoltaic Specialists Conference, Hawaii, 1994, p.1250.

[2] J.R. Davis, Jr., A. Rohatgi, R.H. Hopkins, P.D. Blais, P. Rai-Choudhury, J.R. McCormic, and H.C. Mollenkopf, IEEE Trans. Electron. Devices, ED-27, 1980, p.677.
[3] J.Du Plessis and G.N. Van Wyk, J. Phys. Chem. Solids, 49(12), 1988, p. 1441 and p. 1451.

[4] T.F. Ciszek, in: Semiconductor Silicon 1973, Eds. H.R. Huff and R.R. Bugess (Electrochem. Soc., Pennington, NJ, 1973), p.150. 